\title{
Nontrivalent graph cocycle and cohomology of the long knot space
}

\author{
KEIICHI SAKAI
}

\begin{abstract}
In this paper we show that via the configuration space integral construction a nontrivalent graph cocycle can also yield a nonzero cohomology class of the space of higher (and even) codimensional long knots. This simultaneously proves that the Browder operation induced by the operad action defined by R Budney is not trivial.
\end{abstract}

58D10; 55P48, 81Q30

\section{Introduction}

Recently the (co)homological properties of the spaces $\mathcal{K}_{n}$ (or $\widetilde{\mathcal{K}}_{n}$ ) of (framed) long knots in $\mathbb{R}^{n}$ have been widely studied: the classical case $(n=3)$ by R Budney [3] and Budney and FCohen [4] and the case $n>3$ by D Sinha [14; 16], V Tourtchine [17], P Salvatore [13], P Lambrechts, Tourtchine and I Volić [8] and others. Their approaches in some senses make use of the little disks operad and its action on $\widetilde{\mathcal{K}}_{n}$, which induces on $H_{*}\left(\widetilde{\mathcal{K}}_{n}\right)$ the Browder operation, giving a structure of a Poisson algebra. This Poisson structure has been studied in [13; 17] and by the author in [12], and so on, but is not well understood.

There is another geometric approach to $H_{\mathrm{DR}}^{*}\left(\mathcal{K}_{n}\right)$ (or $\left.H_{\mathrm{DR}}^{*}\left(\widetilde{\mathcal{K}}_{n}\right)\right)$. A Cattaneo, PCottaRamusino and R Longoni [5] constructed a cochain map from certain graph complex to the de Rham complex of $\mathcal{K}_{n}(n>3)$ via perturbative expansion of Chern-Simons theory, which generalizes the integral expression of the Vassiliev invariants for knots in $\mathbb{R}^{3}$ due to R Bott and C Taubes [2] and independently to T Kohno [7]. Moreover they proved that the induced map on cohomology is injective on the trivalent graph cocycles. The injectivity was proved by evaluating the cohomology classes over the cycles obtained from chord diagrams.

Almost nothing is known about the cohomology classes coming from nontrivalent graphs (in the case of ordinary knots, there is a result of Longoni [9]; see below). One reason is that we do not know the corresponding homology cycles.

In this paper we combine the de Rham theory for $\mathcal{K}_{n}(n>3)$ with the action of little disks operad and obtain the first example of a nontrivalent graph cocycle which realizes a nonzero cohomology class of $\mathcal{K}_{n}, n>3$. 
Theorem 1.1 (for notation, see Section 3) Suppose $n>3$ is odd. Then the graph cohomology group $H^{3,1}\left(\mathcal{D}^{*}\right)$ consisting of trivalent graphs with exactly one fourvalent vertex is isomorphic to $\mathbb{R}$. Its generator $\Gamma$ gives a nontrivial element $I(\Gamma) \in$ $H_{\mathrm{DR}}^{3 n-8}\left(\mathcal{K}_{n}\right)$ via the configuration space integral.

Theorem 1.1 is an analogous result to those of Salvatore [13] and the author [12], but the proof is more geometric. We prove the nontriviality of $I(\Gamma)$ by evaluating it on a cycle produced by the action of little disks operad on the space $\widetilde{\mathcal{K}}_{n}$, defined by Budney [3]. Thus we immediately obtain the following.

Corollary 1.2 When $n>3$ is odd, the Browder operation induced by the operad action on $\widetilde{\mathcal{K}}_{n}$ in the sense of [3] is nontrivial.

Remark 1.3 Our cycle represents an element of $H_{3 n-8}\left(\widetilde{\mathcal{K}}_{n}\right)$, the homology of the space of framed long knots. But what we will prove is that its image in $H_{3 n-8}\left(\mathcal{K}_{n}\right)$, the homology of the space of long knots itself, via the map $r: \widetilde{\mathcal{K}}_{n} \rightarrow \mathcal{K}_{n}$ (see Lemma 2.2) does not vanish.

The cohomology classes of $\mathcal{K}_{n}$ obtained from trivalent graphs can be seen as "higher dimensional analogues" of the finite type invariants for knots in $\mathbb{R}^{3}$. But the cohomology class obtained in Theorem 1.1 is not such a one. It would be an interesting problem to determine where the cohomology class of $\mathcal{K}_{3}$ corresponding to the class obtained in Theorem 1.1 (if it exists) fits in the recursive formula (see Budney and Cohen [4]) for the homotopy type of components of $\mathcal{K}_{3}$.

When the first version of this paper was submitted, the author was not aware of Longoni's result [9] for the space $\operatorname{Emb}\left(S^{1}, \mathbb{R}^{n}\right)$ of closed (ordinary) knots in $\mathbb{R}^{n}$. Longoni found a nontrivalent graph cocycle (different from ours) when $n>3$ is even and made a nonzero element of $H^{3(n-3)+1}\left(\operatorname{Emb}\left(S^{1}, \mathbb{R}^{n}\right)\right)$ from the cocycle. The proof is also similar to ours, that is, the evaluation of the cocycle on the dual cycle. But the construction of the cycle naturally differs from ours, since there is no operad action on Emb $\left(S^{1}, \mathbb{R}^{n}\right)$. Longoni's cycle is "secondarily" defined by using 4-term relations, while we use an operad action.

This paper is organized as follows. In the Section 2 we recall the action of little disks operad on the space of framed knots, following Budney [3], and construct a cycle on which our cocycle will be evaluated. Section 3 is devoted to reviewing the configuration space integral. The readers familiar with the construction due to Cattaneo, Cotta-Ramusino and Longoni [5] may skip this section, except for Section 3.4 where our nontrivalent graph cocycle is given. In Section 4 we will prove Theorem 1.1 and give a brief comment on the further computation on $H_{\mathrm{DR}}^{k(n-3)+1}\left(\mathcal{K}_{n}\right), k \geq 4$. 
Acknowledgements The author expresses his appreciation to Alberto Cattaneo, Ryan Budney, Fred Cohen, Toshitake Kohno, Paolo Salvatore, Victor Tourtchine and the referee for reading the draft of this paper and giving him much useful advice. He is also grateful to Ismar Volić for pointing out a mistake in the previous version of this paper. The author noticed a result of Longoni by virtue of information on Dai Tamaki's website (which is written in Japanese). This research is partially supported by $21 \mathrm{st}$ century COE program at Graduate School of Mathematical Sciences, University of Tokyo.

\section{The space of long knots and little disks action}

\subsection{The space of long knots}

In this paper we always assume $n>3$ is odd.

Definition 2.1 A long knot in dimension $n$ is an embedding

$$
f: \mathbb{R}^{1} \hookrightarrow \mathbb{R}^{n}
$$

such that $f(t)=(0, \ldots, 0, t)$ if $|t| \geq 1$.

Denote the unit ball in $\mathbb{R}^{m}$ by $B^{m}$;

$$
B^{m}:=\left\{x \in \mathbb{R}^{m}|| x \mid \leq 1\right\} .
$$

A framed long knot in dimension $n$ is an embedding

$$
g: B^{n-1} \times \mathbb{R}^{1} \hookrightarrow B^{n-1} \times \mathbb{R}^{1}
$$

such that $g(x, t)=(x, t)$ if $|t| \geq 1$. Denote the space of all (framed) long knots in $\mathbb{R}^{n}$ by $\mathcal{K}_{n}$ (respectively $\widetilde{\mathcal{K}}_{n}$ ).

The space $\widetilde{\mathcal{K}}_{n}$ defined as above was denoted by $E C\left(1, B^{n-1}\right)$ by Budney [3]. We define the framed long knots as in the cylinder, because it becomes easier in this setting to define the little disks action.

We have a forgetting map $r: \widetilde{\mathcal{K}}_{n} \rightarrow \mathcal{K}_{n}$ defined by

$$
r(f)(t)=f(0, t), \quad \forall f \in \widetilde{\mathcal{K}}_{n} .
$$

Lemma 2.2 [3] The map $r$ is equivalent to a trivial fibration with fiber $\Omega S O(n-1)$. Hence $\widetilde{\mathcal{K}}_{n} \simeq \mathcal{K}_{n} \times \Omega S O(n-1)$. 


\subsection{Some cycles}

The subgroup $\bigoplus_{k \geq 0} H_{(n-3) k}\left(\mathcal{K}_{n}\right)$ is known to be nontrivial (see Cattaneo, CottaRamusino and Longoni [5], Longoni [9], Sinha [16] and Tourtchine [17]) since it contains the subalgebra isomorphic to the algebra $\mathcal{A}$ of chord diagrams modulo 4-term and 1-term relations. Few other cycles are known. The purpose of this paper is to find another (co)cycle which does not come from any chord diagrams.

Here we explain two examples of cycles made from chord diagrams, namely, $e \in$ $H_{n-3}\left(\widetilde{\mathcal{K}}_{n}\right)$ and $v_{2} \in H_{2(n-3)}\left(\mathcal{K}_{n}\right)$ which we will use later. More general treatments can be found in $[5 ; 9 ; 12 ; 17]$.

2.2.1 The cycle $v_{2}$ Consider the chord diagram $V$ in Figure 1, which is thought of as corresponding to an immersion $f$ with two transversal doublepoints $z_{i}=f\left(\xi_{i}\right)=$ $f\left(\xi_{i+2}\right), i=1,2, \xi_{1}<\xi_{2}<\xi_{3}<\xi_{4}$, see Figure 2. Since we assume $n>3$, the immersion $f$ is determined uniquely up to homotopy.

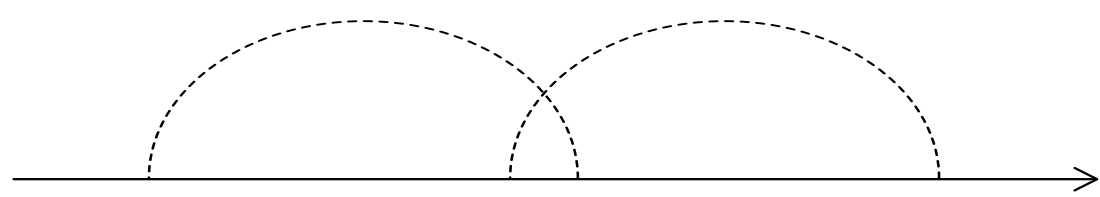

Figure 1: Chord diagram $V$

At each self-intersections $z_{i}$ we have resolutions of $f$ parametrized by $S^{n-3}$ (see Figure 3), so we obtain a map

$$
\alpha(V):\left(S^{n-3}\right)^{2} \longrightarrow \mathcal{K}_{n} .
$$

More explicitly, the knot $\alpha(V)\left(u_{1}, u_{2}\right)$ is defined in [5; 9] by

$$
\alpha(V)\left(u_{1}, u_{2}\right)(t)= \begin{cases}f(t)+\delta_{i} u_{i} \exp \left(\frac{1}{\left(t-\xi_{i}\right)^{2}-\varepsilon_{i}^{2}}\right) & \left|t-\xi_{i}\right|<\varepsilon_{i}, i=1,2 \\ f(t) & \text { otherwise }\end{cases}
$$

where $\delta_{i}$ and $\varepsilon_{i}$ are small positive numbers, and $u_{i} \in S^{n-3}$ is realized as a unit vector in $\mathbb{R}^{n}$ which is perpendicular to $f^{\prime}\left(\xi_{i}\right)$ and $f^{\prime}\left(\xi_{i+2}\right)$.

Remark 2.3 [5] All the resolutions generate $S^{n-2}$;

$$
S_{i}:=\bigcup_{u_{i} \in S^{n-3}} \bigcup_{\left|t-\xi_{i}\right|<\varepsilon_{i}} \alpha(V)\left(u_{1}, u_{2}\right)(t) \approx S^{n-2},
$$



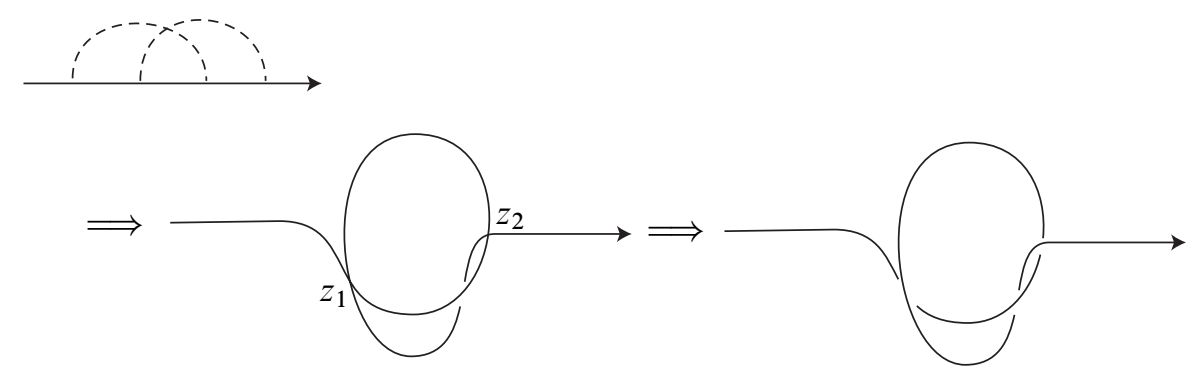

Figure 2: The correspondence $V \mapsto f_{V} \mapsto \alpha(V)$
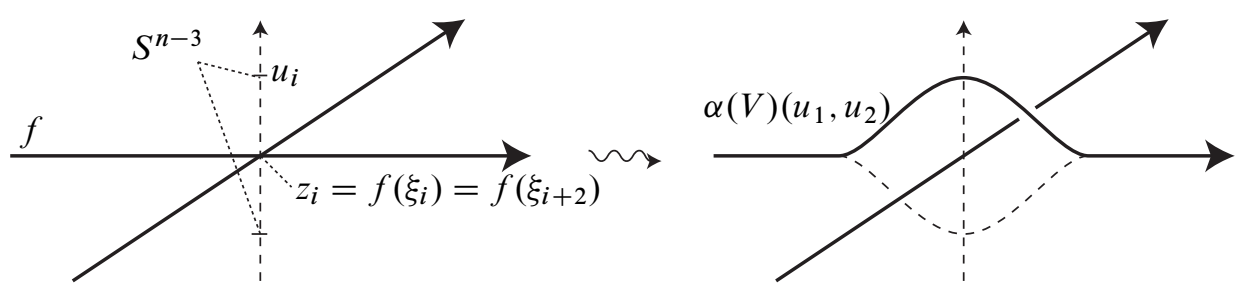

Figure 3: Resolution of the self-intersection

which has the linking number one with the segment $I_{i}:=f\left(\xi_{i+2}-\varepsilon_{i}, \xi_{i+2}+\varepsilon_{i}\right)$, $i=1,2$ (see Figure 3).

We regard the map $\alpha(V)$ as a $2(n-3)-$ cycle of $\mathcal{K}_{n}$, and denote its homology class by $v_{2} \in H_{2(n-3)}\left(\mathcal{K}_{n}\right)$ (because it can be seen as a dual of the order two invariant for knots in $\mathbb{R}^{3}$; see Bott and Taubes [2] and Kohno [7]).

This construction extends to general chord diagrams.

Proposition $2.4 \quad[5 ; 9 ; 12 ; 17]$ The correspondence $\Gamma \mapsto \alpha(\Gamma)$ is defined for any chord diagrams and determines an injective homomorphism of algebras

$$
\alpha: \mathcal{A} \longrightarrow \bigoplus_{k \geq 0} H_{k(n-3)}\left(\mathcal{K}_{n}\right),
$$

where $\mathcal{A}$ is an algebra generated by chord diagrams modulo 4-term relations (see Figure 4) and 1-term relation, that is, a chord diagram with an isolated chord (a chord which does not intersect with other chords) is regarded as zero. The product of $\mathcal{A}$ is defined as the concatenation of diagrams, while that of $H_{*}\left(\mathcal{K}_{n}\right)$ is induced by the connecting sum (see Figure 5). 


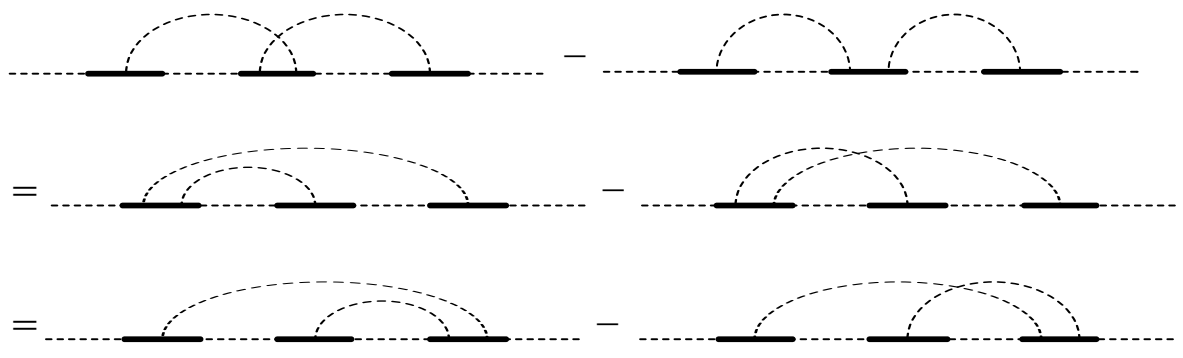

Figure 4: 4-term relations

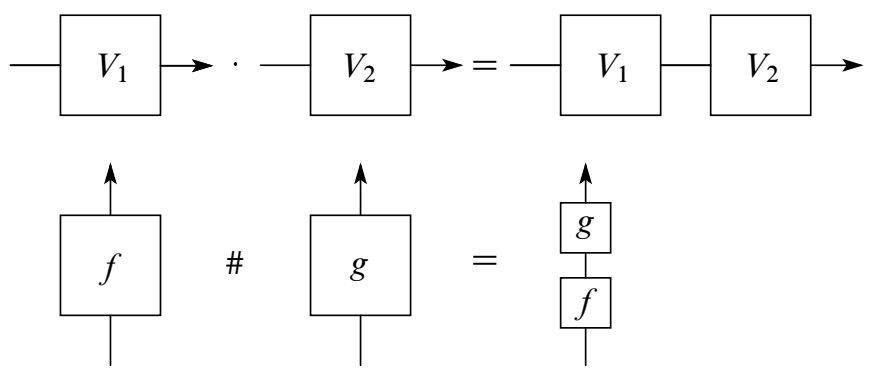

Figure 5: Product of two chord diagrams $V_{1}, V_{2}$, and connect sum of two long knots $f, g$

2.2.2 The cycle $e \quad$ A resolution of an isolated chord yields a null-homologous cycle of $\mathcal{K}_{n}$ (recall the Reidemeister move I for knots in $\mathbb{R}^{3}$ ). Instead we assign a homology cycle $e \in H_{n-3}(\Omega S O(n-1))$ to $\Gamma_{0}$, a chord diagram with only one chord. First consider the cycle $e^{\prime}$ of $S O(n-1)$ realized by a map

$$
e^{\prime}: \Sigma S^{n-3} \longrightarrow S O(n-1),
$$

called "the clutching map for the tangent bundle of $S^{n-1}$," defined below; where the suspension $\Sigma S^{n-3} \approx S^{n-2}$ is defined by collapsing the subsets $(-\infty,-1] \times S^{n-3}$ and $[1, \infty) \times S^{n-3}$ of $\mathbb{R}^{1} \times S^{n-3}$ to points. We think of $S^{n-3}$ as a unit sphere in $\mathbb{R}^{n-2}$ and $\Sigma S^{n-3}$ as in $\mathbb{R}^{n-1}$, by using an inclusion $\Sigma S^{n-3} \rightarrow \mathbb{R}^{n-2} \times \mathbb{R}^{1}$,

$$
[s, u] \longmapsto \begin{cases}\left(\sqrt{1-s^{2}} u, s\right) & s \in[-1,1], \\ (0,-1) & s \leq-1, \\ (0,1) & s \geq 1 .\end{cases}
$$


The map $e^{\prime}: \Sigma S^{n-3} \rightarrow S O(n-1)$ is defined by

$$
e^{\prime}[s, u]=H_{x_{n-1}} H_{[s, u]},
$$

where $H_{[s, u]} \in O(n-1)$ is the reflection with respect to the orthogonal complement of $[s, u] \in \mathbb{R}^{n-1}\left(H_{x_{n-1}}\right.$ is the reflection with respect to the hyperplane $\left.\left\{x_{n-1}=0\right\}\right)$. Since $e^{\prime}(1, u)=e^{\prime}(-1, u)=I_{n-1}$, the adjoint map

$$
e: S^{n-3} \longrightarrow \Omega S O(n-1), \quad e(u)(s):=e^{\prime}[s, u]
$$

to $e^{\prime}$ is defined and represents the desired cycle $[e]$. It is known that $[e]$ determines nontrivial homology class only if $n$ is odd.

We regard $e \in H_{n-3}\left(\widetilde{\mathcal{K}}_{n}\right)$ by composing $e$ with $j: \Omega S O(n-1) \hookrightarrow \widetilde{\mathcal{K}}_{n}$ defined by

$$
j(\gamma)(x, t)=(\gamma(t) x, t),
$$

here $\gamma(t) \in S O(n-1)$ is seen as a linear transformation in $\mathbb{R}^{n-1} \times\{0\}$.

We have described two cycles $e$ and $v_{2}$. Below we will show that the Poisson bracket $\lambda\left(e, v_{2}\right)$ is not zero. The Poisson structure is induced from an action of little disks operad, and will be explained in the next subsection.

\subsection{Little disks action}

Definition 2.5 A little $m$-ball is an embedding $b: B^{m} \hookrightarrow B^{m}$ of the form

$$
b(x)=r(x-p)
$$

for some $p \in B^{m}$ and $0<r \leq 1$. Define the little $m$-balls operad $\mathcal{B}_{m}$ by setting

$$
\mathcal{B}_{m}(k):=\left\{\begin{array}{l|c}
\left(b_{1}, \ldots, b_{k}\right) & \begin{array}{c}
b_{i} \text { a little } m \text {-ball, } \\
b_{i}\left(\operatorname{Int} B^{m}\right) \cap b_{j}\left(\operatorname{Int} B^{m}\right)=\varnothing \text { if } i \neq j
\end{array}
\end{array}\right\}
$$

for $k \geq 1$. The operad structure is defined in a familiar way (see May [10]).

Here we recall the operad action of $\mathcal{B}_{2}$ on $\widetilde{\mathcal{K}}_{n}$ defined by Budney [3], that is, the "associative" maps

$$
\kappa(k): \mathcal{B}_{2}(k) \times\left(\widetilde{\mathcal{K}}_{n}\right)^{k} \longrightarrow \widetilde{\mathcal{K}}_{n}, \quad k \geq 1 .
$$

Given $b=\left(b_{1}, \ldots, b_{k}\right) \in \mathcal{B}_{2}(k)$, consider the projections

$$
I_{j}:=\operatorname{pr}_{1} \circ b_{j}\left(B^{2}\right) \subset[-1,1], \quad 1 \leq j \leq k .
$$

There are the little $1-$ balls $l_{j}(t)=a_{j} t+b_{j}$ such that $l_{j}([-1,1])=I_{j}, 1 \leq j \leq k$ $\left(l_{1}, \ldots, l_{k}\right.$ are not necessarily disjoint mutually). 
A little 1 -ball $l:[-1,1] \rightarrow[-1,1], l(t)=a t+b$, extends to a diffeomorphism $\tilde{l}: \mathbb{R}^{1} \rightarrow \mathbb{R}^{1}$ in an obvious way, and determines a map

$$
\begin{gathered}
\mu_{l}: \widetilde{\mathcal{K}}_{n} \longrightarrow \widetilde{\mathcal{K}}_{n}, \\
\mu_{l}(f):=\left(\operatorname{id}_{B^{n-1}} \times \tilde{l}\right) \circ f \circ\left(\operatorname{id}_{B^{n-1}} \times \tilde{l}^{-1}\right) .
\end{gathered}
$$

For any little $2-$ ball $b$, define the number $t_{b} \in[-1,1]$ by

$$
t_{b}=\min \left\{y \mid(x, y) \in b\left(B^{2}\right) \text { for some } x\right\} .
$$

With this notation in hand, we can define the map $\kappa$ by

$$
\kappa(k)\left(\left(b_{1}, \ldots, b_{k}\right) ;\left(f_{1}, \ldots, f_{k}\right)\right):=\mu_{l_{\sigma(1)}}\left(f_{\sigma(1)}\right) \circ \cdots \circ \mu_{l_{\sigma(k)}}\left(f_{\sigma(k)}\right),
$$

where $\sigma \in \mathfrak{S}_{k}$ is such that $t_{b_{\sigma(1)}} \leq \cdots \leq t_{b_{\sigma(k)}}$.

Theorem 2.6 [3] The maps $\kappa(k)(k \geq 1)$ are well defined and defines an action of the operad $\mathcal{B}_{2}$ on $\widetilde{\mathcal{K}}_{n}$.

In particular, $\kappa(2): \mathcal{B}_{2}(2) \times\left(\widetilde{\mathcal{K}}_{n}\right)^{2} \rightarrow \widetilde{\mathcal{K}}_{n}$ is "pushing one long knot $f_{1}$ through another long knot $f_{2}$, afterward pushing $f_{2}$ through $f_{1}$ " (see Figure 6 and [3, Figures $\left.2,5,7\right]$ ).
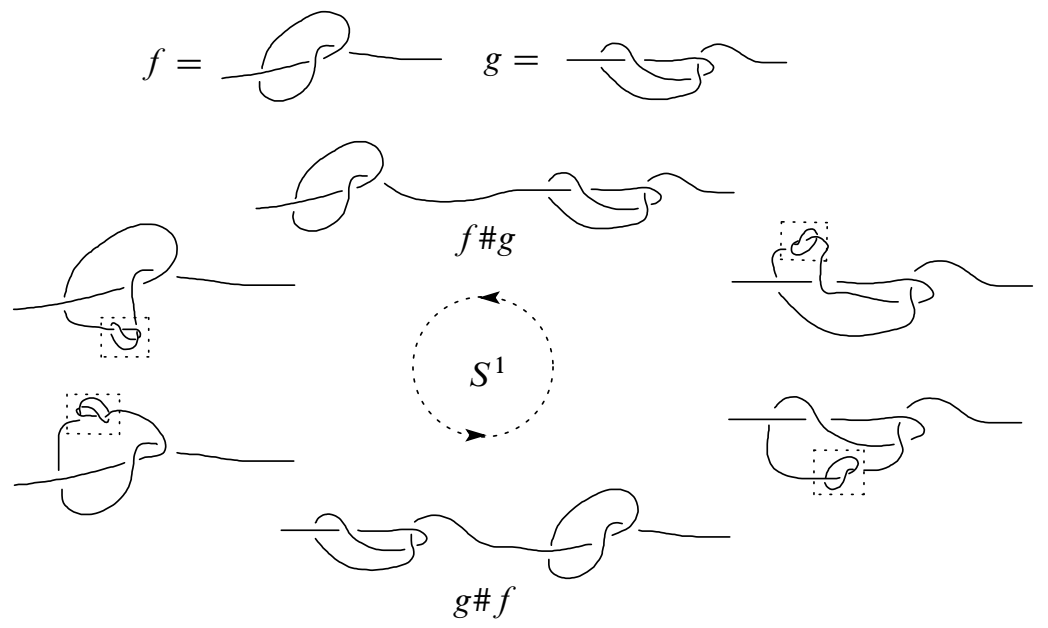

Figure 6: A picture of $\kappa(2)$; notice that $\mathcal{B}_{2}(2) \simeq S^{1}$. 
The space $\mathcal{B}_{2}(2)$ is homotopy equivalent to $S^{1}$. The map $\kappa(2): \mathcal{B}_{2}(2) \times\left(\widetilde{\mathcal{K}}_{n}\right)^{2} \rightarrow \widetilde{\mathcal{K}}_{n}$ induces on homology two products

$$
\begin{aligned}
& *: H_{p}\left(\widetilde{\mathcal{K}}_{n}\right) \otimes H_{q}\left(\widetilde{\mathcal{K}}_{n}\right) \longrightarrow H_{p+q}\left(\widetilde{\mathcal{K}}_{n}\right), \\
& \lambda: H_{p}\left(\widetilde{\mathcal{K}}_{n}\right) \otimes H_{q}\left(\widetilde{\mathcal{K}}_{n}\right) \longrightarrow H_{p+q+1}\left(\widetilde{\mathcal{K}}_{n}\right)
\end{aligned}
$$

corresponding to generators of $H_{p}\left(\mathcal{B}_{2}(2)\right), p=0,1$, respectively. The former product is equal to that induced by the connecting sum. The latter $\lambda$ is called Browder operation and is a 1-Poisson bracket, that is, a Lie bracket of degree one, satisfying the Leibniz rule (see Cohen [6]).

Our attention will be paid to the element $\lambda\left(e, v_{2}\right) \in H_{3 n-8}\left(\widetilde{\mathcal{K}}_{n}\right)$ or its image $\Lambda:=$ $r_{*} \lambda\left(e, v_{2}\right) \in H_{3 n-8}\left(\mathcal{K}_{n}\right)$ via the forgetful map $r: \widetilde{\mathcal{K}}_{n} \rightarrow \mathcal{K}_{n}$. For definiteness, we choose a map

$$
v:\left(S^{n-3}\right)^{2} \longrightarrow \mathcal{K}_{n}
$$

representing $v_{2}$ by resolving an immersion $f$ (Figure 7). Most part of the embedding lies in the $x_{n-1} x_{n}$-plane. The self-intersections to be resolved are $z_{i}=f\left(\xi_{i}\right)=$ $f\left(\xi_{i+2}\right), \xi_{i}<\xi_{i+2}, i=1,2$. The vectors $u_{i} \in S^{n-3}, i=1,2$ (which are normal to $x_{n-1} x_{n}$-plane) produce the resolutions of the self-intersections $z_{i}$, respectively. The segments $l$ are included in the $x_{n}$-axis.

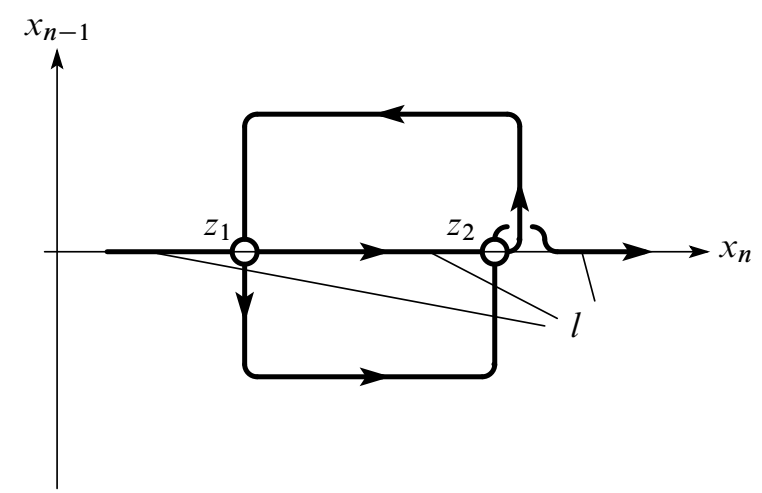

Figure 7: The cycle $v_{2}$

Given the "trivial frame," $v$ can represent the cycle $[v]=v_{2} \in H_{2(n-3)}\left(\widetilde{\mathcal{K}}_{n}\right)$.

Then the representative $\lambda(e, v)$ is the family of embeddings defined by "pushing $e$ through $v$, afterward pushing $v$ through $e . "$

We want to know the representative of $\Lambda=r_{*}\left(\lambda\left(e, v_{2}\right)\right) \in H_{3 n-8}\left(\mathcal{K}_{n}\right) . \Lambda$ is obtained from $\lambda(e, v)$ by forgetting the frame. When $e$ goes through $v$, the frame of $v$ "near" 
$e$ would be agitated. But this phenomenon disappears after forgetting the frame via $r: \widetilde{\mathcal{K}}_{n} \rightarrow \mathcal{K}_{n}$. In contrast, when $v$ passes through $e$, the whole embedding $v$ "rotates" around $x_{n}$-axis via the frame $e$, and this phenomenon does not disappear even if we forget the frame. Thus $\Lambda$ is represented by " $v$ rotated by $e . "$

More precisely, if we think of $S O(n-1)$ as a subgroup of $S O(n)$ fixing the $x_{n}$-axis, then $\Lambda$ can be represented by the map

$$
\begin{gathered}
\Lambda: \Sigma S^{n-3} \times\left(S^{n-3}\right)^{2} \longrightarrow \mathcal{K}_{n}, \\
\Lambda\left(\left[s, u_{0}\right], u_{1}, u_{2}\right)(t):=e\left(\left[2 s+p\left(v\left(u_{1}, u_{2}\right)(t)\right), u_{0}\right]\right) v\left(u_{1}, u_{2}\right)(t), \quad t \in \mathbb{R}^{1},
\end{gathered}
$$

where $p: \mathbb{R}^{n} \rightarrow \mathbb{R}^{1}$ is the projection $\left(x_{1}, \ldots, x_{n}\right) \mapsto x_{n}$. Thus $\Lambda\left(\left[s, u_{0}\right], u_{1}, u_{2}\right)$ is a long knot $v\left(u_{1}, u_{2}\right)$ with its intersection with $p^{-1}(a)$ being rotated around the $x_{n}$-axis by the frame $e\left[2 s+a, u_{0}\right] \in S O(n-1),|a| \leq 1$.

The cycle $\Lambda$ has a simpler description; for $0 \leq \tau \leq 1$, define

$$
\begin{gathered}
\Lambda_{\tau}^{\prime}: \Sigma S^{n-3} \times\left(S^{n-3}\right)^{2} \longrightarrow \mathcal{K}_{n}, \\
\Lambda_{\tau}^{\prime}\left(\left[s, u_{0}\right], u_{1}, u_{2}\right)(t):=e\left(\left[(2-\tau) s+(1-\tau) p\left(v\left(u_{1}, u_{2}\right)(t)\right), u_{0}\right]\right) v\left(u_{1}, u_{2}\right)(t),
\end{gathered}
$$

then $\Lambda_{\tau}^{\prime}$ is well defined for any $\tau \in[0,1], \Lambda_{0}^{\prime}=\Lambda$ and

$$
\Lambda_{1}^{\prime}\left(\left[s, u_{0}\right], u_{1}, u_{2}\right)(t)=e\left(\left[s, u_{0}\right]\right) v\left(u_{1}, u_{2}\right)(t) .
$$

Below we rewrite $\Lambda:=\left[\Lambda_{1}^{\prime}\right] \in H_{3 n-8}\left(\mathcal{K}_{n}\right)$. This $\Lambda$ is $v\left(u_{1}, u_{2}\right)$ rotated all together by $e\left[s, u_{0}\right]$.

Remark 2.7 There are several ways to define the action of $\mathcal{B}_{2}$. In [16] D Sinha constructed a cosimplicial model for the space $\mathcal{K}_{n}^{\prime}$ of "long knots modulo immersions," a space which relates to $\widetilde{\mathcal{K}}_{n}$, and proved that the space is a little disks object by means of McClure-Smith machinery [11]. It can be proved [13; 12] that, when $n>3$ is odd, the induced Browder operation is not zero;

$$
\lambda: H_{n-3}\left(\mathcal{K}_{n}^{\prime}, \mathbb{R}\right) \otimes H_{2(n-3)}\left(\mathcal{K}_{n}^{\prime}, \mathbb{R}\right) \stackrel{\cong}{\rightrightarrows} H_{3 n-8}\left(\mathcal{K}_{n}^{\prime}, \mathbb{R}\right) .
$$

It is still unknown how the operad actions on $\widetilde{\mathcal{K}}_{n}$ and $\mathcal{K}_{n}^{\prime}$ relate to each other. So Corollary 1.2 is the first result about the nontriviality of the Browder operation in the sense of Budney [3] in higher codimensional case (when $n=3$, the Browder operation is highly nontrivial; see Budney and Cohen [4]). 


\section{Configuration space integral}

Here we recall the main result of Cattaneo, Cotta-Ramusino and Longoni [5] when $n>3$ is odd (this paper also treats the even-dimensional case). Readers can also refer to Bott and Taubes [2], Kohno [7] and Volić [18].

\subsection{Graph complex}

Definition 3.1 [5] Our graph consists of the following data.

(1) Any graph has an oriented line called the special line.

(2) A graph has two types of vertices (the set of vertices is possibly empty); those on the special line and those not on the line. In [18] the former vertices are called interval ones, while the latter free. The vertices are labeled by $1,2, \ldots, m$ for an appropriate $m \geq 0$ so that the labels of the interval vertices are smaller than those of free vertices.

(3) Vertices are connected by oriented edges so that the graph is connected. The valency of each vertex is at least three. An edge may have only one interval vertex as its endpoints (such an edge is called a small loop).

(4) If an edge $e$ is a small loop at the interval vertex, then we give the order of the half-edges of $e$ (which is defined independently of the orientation of $e$ ).

Let $\Gamma$ be a graph with $e$ edges, $v_{i}$ interval vertices and $v_{f}$ free vertices. Define

$$
\begin{aligned}
& \operatorname{ord} \Gamma:=e-v_{f}, \\
& \operatorname{deg} \Gamma:=2 e-3 v_{f}-v_{i} .
\end{aligned}
$$

An example of a graph is shown in Figure 8.

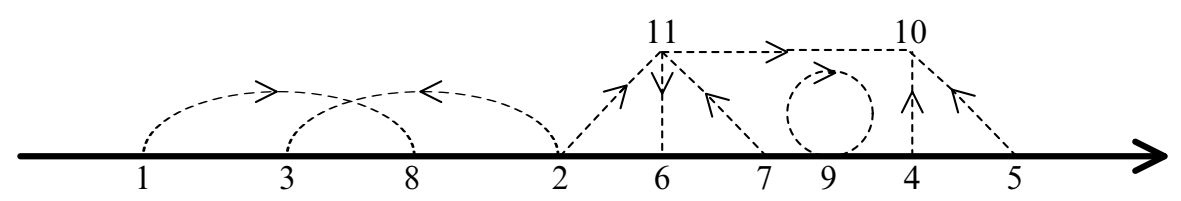

Figure 8: An element of $\mathcal{D}^{7,3}$

Remark 3.2 For any graph $\Gamma$, its order and degree are not less than zero. One can easily prove that $1-$ ord $\Gamma$ is equal to the Euler characteristic of the one dimensional $\mathrm{CW}$-complex $\Gamma$, and that deg $\Gamma$ is zero if and only if $\Gamma$ is a trivalent graph. 
Consider the vector space spanned by the graphs with ord $\Gamma=k$ and $\operatorname{deg} \Gamma=l$ modulo the subspace generated by

(1) $\Gamma$, two vertices of which are joined by more than one edges,

(2) $\Gamma$ with a small loop whose endpoint is a free vertex, and

(3) $\Gamma^{\prime}-(-1)^{\operatorname{sign} \sigma} \Gamma$, here $\Gamma^{\prime}$ is obtained from $\Gamma$ by a permutation $\sigma$ which permutes the labels of the vertices (so that the labels of the interval vertices are less than those of free vertices) or reversing the orientations of the edges.

We denote the quotient space by $\mathcal{D}^{k, l}$.

The differential $\delta: \mathcal{D}^{k, l} \rightarrow \mathcal{D}^{k, l+1}$ is defined as follows. For any graph $\Gamma, \delta \Gamma$ is the signed sum of graphs obtained by contracting, one at a time, the edges one of whose endpoint is not an interval vertex, and the arcs, portions of the special line bounded by two consecutive interval vertices.

Determining precisely the labels and signs of the graphs after contraction [5], we can show the following directly by definition.

Theorem 3.3 [5] The map $\delta$ sends $\mathcal{D}^{k, l}$ to $\mathcal{D}^{k, l+1}$, and $\delta^{2}=0$.

Example 3.4 Two examples of $\delta: \mathcal{D}^{2,0} \rightarrow \mathcal{D}^{2,1}$ are given in Figure 9, which shows

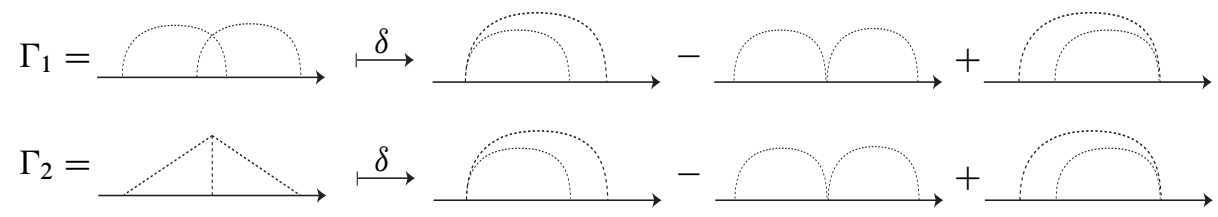

Figure 9: Examples of the coboundary operator

that a cochain $\Gamma_{1}-\Gamma_{2}$ is a cocycle in $\mathcal{D}^{2,0}$.

\subsection{Configuration space integrals}

Below we will associate a differential form of $\mathcal{K}_{n}$ with a given graph $\Gamma$.

We denote the configuration space by

$$
\operatorname{Conf}(X, m):=\left\{\left(x_{1}, \ldots, x_{m}\right) \in X^{m} \mid x_{i} \neq x_{j}\right\} .
$$


For any $N$, the Gauss maps $\varphi_{i j}: \operatorname{Conf}\left(\mathbb{R}^{N}, m\right) \rightarrow S^{N-1}(1 \leq i \neq j \leq m)$ are defined by

$$
\varphi_{i j}\left(x_{1}, \ldots, x_{m}\right)=\frac{x_{i}-x_{j}}{\left|x_{i}-x_{j}\right|} .
$$

We use the compactifications of the configuration spaces.

Theorem 3.5 (Axelrod and Singer [1], Bott and Taubes [2] and Sinha [15]) For any manifold $M$, we can construct a compact manifold $\operatorname{Conf}[M, m]$ with corners, which is a compactification of $\operatorname{Conf}(M, m)$ in the sense that the interior of $\operatorname{Conf}[M, m]$ is $\operatorname{Conf}(M, m)$. When $M=\mathbb{R}^{N}$, then the Gauss maps $\varphi_{i j}$ can be extended smoothly onto the boundary of $\operatorname{Conf}\left[\mathbb{R}^{N}, m\right]$.

Roughly speaking, the points in Conf $[M, m]$ may "collide with each other," but in such cases, information of the directions of the collision must be recorded.

Let $\Gamma \in \mathcal{D}^{k, l}$ be a graph with $e$ edges, $v_{i}$ interval vertices and $v_{f}$ free vertices (hence $e-v_{f}=k, 2 e-3 v_{f}-v_{i}=l$ ). Consider the following pullback square:

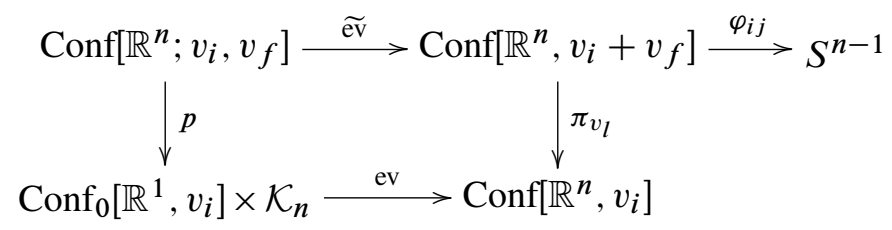

Here $\operatorname{Conf}_{0}\left[\mathbb{R}^{1}, m\right]$ is a connected component corresponding to $t_{1} \leq t_{2} \leq \cdots \leq t_{m}$ and, on the interior, ev and $\pi_{*}$ are defined by

$$
\begin{aligned}
\operatorname{ev}\left(\left(t_{1}, \ldots, t_{v_{i}}\right), f\right) & :=\left(f\left(t_{1}\right), \ldots, f\left(t_{v_{i}}\right)\right), \\
\pi_{v_{i}}\left(x_{1}, \ldots, x_{v_{i}+v_{f}}\right) & :=\left(x_{1}, \ldots, x_{v_{i}}\right) .
\end{aligned}
$$

$\operatorname{Conf}\left[\mathbb{R}^{n} ; v_{i}, v_{f}\right]$ is the space of pairs $\left(\left(x_{1}, \ldots, x_{v_{i}+v_{f}}\right), f\right)$, where

$$
\left(x_{1}, \ldots, x_{v_{i}+v_{f}}\right) \in \operatorname{Conf}\left[\mathbb{R}^{n}, v_{i}+v_{f}\right]
$$

and $f \in \mathcal{K}_{n}$, with $x_{1}, \ldots, x_{v_{i}}$ on the knot $f$.

With an edge (or a small loop) $\overrightarrow{i j}$ of $\Gamma$, we assign a differential form

defined by

$$
\begin{array}{r}
\theta_{i j} \in \Omega^{n-1}\left(\operatorname{Conf}\left[\mathbb{R}^{n} ; v_{i}, v_{f}\right]\right) \\
\theta_{i j}:= \begin{cases}\widetilde{\operatorname{ev}}^{*} \varphi_{i j}^{*} \operatorname{vol}_{S^{n-1}} & i \neq j, \\
D_{i}^{*} \operatorname{vol}_{S^{n-1}} & i=j .\end{cases}
\end{array}
$$


Here, for $1 \leq i \leq v_{i}$,

$$
\begin{gathered}
D_{i}: \operatorname{Conf}\left[B^{n-1} \times \mathbb{R}^{1} ; v_{i}, v_{f}\right] \longrightarrow S^{n-1}, \\
D_{i}\left(f\left(t_{1}\right), \ldots, f\left(t_{v_{i}}\right), x_{1}, \ldots, x_{v_{f}}\right)=\frac{f^{\prime}\left(t_{i}\right)}{\left|f^{\prime}\left(t_{i}\right)\right|} .
\end{gathered}
$$

We define a form $\theta_{\Gamma} \in \Omega^{(n-1) e}\left(\operatorname{Conf}\left[\mathbb{R}^{n} ; v_{i}, v_{f}\right]\right)$ by

$$
\theta_{\Gamma}:=\bigwedge_{\text {edges } \overrightarrow{i j} \text { of } \Gamma} \theta_{i j} .
$$

Note that this form is defined independently of the order of $\theta_{i j}$ 's, since they are even forms.

Integrating this form along the fiber of

$$
\operatorname{Conf}\left[\mathbb{R}^{n} ; v_{i}, v_{f}\right] \stackrel{p}{\longrightarrow} \operatorname{Conf}_{0}\left[\mathbb{R}^{1}, v_{i}\right] \times \mathcal{K}_{n} \stackrel{\mathrm{pr}_{2}}{\longrightarrow} \mathcal{K}_{n},
$$

we obtain a differential form

$$
I(\Gamma):=\left(\operatorname{pr}_{2} \circ p\right)_{*} \theta_{\Gamma} \in \Omega^{*}\left(\mathcal{K}_{n}\right) .
$$

This integral converges since we compactify the configuration spaces. The degree of the form $I(\Gamma)$ is

$$
\begin{aligned}
(n-1) e-n v_{f}-v_{i} & =(n-3)\left(e-v_{f}\right)+2 e-3 v_{f}-v_{i} \\
& =(n-3) k+l
\end{aligned}
$$

Thus we have a map

$$
I: \mathcal{D}^{k, l} \longrightarrow \Omega^{(n-3) k+l}\left(\mathcal{K}_{n}\right)
$$

Theorem 3.6 [5] If $n>3$ is odd, then the above map I is a cochain map.

Outline of proof By Stokes' theorem, the differential $d I(\Gamma)$ is an integration along the boundary of Conf $\left[\mathbb{R}^{n} ; v_{i}, v_{f}\right]$. Recall that the boundary of Conf $\left[\mathbb{R}^{n} ; v_{i}, v_{f}\right]$ is stratified via the "complexities of degenerations of the configurations" (see Axelrod and Singer [1]). The codimension one strata correspond to the simultaneous collisions of points. We can see $[2 ; 5 ; 18]$ that, when $n>3$, only the "principal faces" (corresponding to the collisions of exactly two points) contribute to the integration $d I(\Gamma)$. These collisions exactly correspond to the differential $\delta$ of the graph complex $\mathcal{D}^{*}$, hence $d I(\Gamma)=I(\delta \Gamma)$. 


\subsection{Trivalent graph cocycles}

Notice that a chord diagram with $k$ chords is thought of as in $\mathcal{D}^{k, 0}$ if some orientation of edges are given to $\Gamma$. The chord diagram has $e=k$ edges, $v_{i}=2 k$ interval vertices, and no free vertices $\left(v_{f}=0\right)$.

The proof of the following is a combinatorial one.

Lemma 3.7 [5] Let $\Gamma=\sum_{i} a_{i} \Gamma_{i} \in \mathcal{D}^{k, 0}$ be a nonzero cocycle with each $\Gamma_{i}$ trivalent graphs. Then there is at least one graph, say $\Gamma_{1}$, which is a chord diagram. Moreover, all the chord diagrams contained in the summand of $\Gamma$ has no isolated chord.

For example, a cochain $\Gamma_{1}-\Gamma_{2}$ given in Example 3.4 contains a chord diagram $\Gamma_{1}$, and there is no isolated chord.

Let $\Gamma=\sum_{i} a_{i} \Gamma_{i} \in \mathcal{D}^{k, 0}$ be a nonzero cocycle, and suppose $\Gamma_{1}$ is a chord diagram with $a_{1} \neq 0$. Then $I(\Gamma) \in H_{\mathrm{DR}}^{(n-3) k}\left(\mathcal{K}_{n}\right)$ turns out to be not zero by the following theorem.

Theorem 3.8 [5] Denote by $\langle$,$\rangle the pairing of cocycles with cycles. Then we have$ $\left\langle I(\Gamma), \alpha\left(\Gamma_{1}\right)\right\rangle= \pm a_{1}$.

A detailed proof can be found in [5]. Here, as an example, we compute $\left\langle I(\Gamma), v_{2}\right\rangle$ where $\Gamma=\Gamma_{1}-\Gamma_{2}$ is a cocycle given in Example 3.4 (notice that the cycle $v_{2}$ is equal to $\alpha\left(\Gamma_{1}\right)$ ). This computation is easily generalized to prove Theorem 3.8, and gives us a lot of useful suggestions for the proof of our main theorem.

Let $v_{i}$ and $v_{f}$ the numbers of interval and free vertices of the graph $\Gamma_{j}, j=1,2$ (if $j=1$, then $v_{i}=4$ and $v_{f}=0$; if $j=2$, then $v_{i}=3, v_{f}=1$ ). Consider the following pullback square:

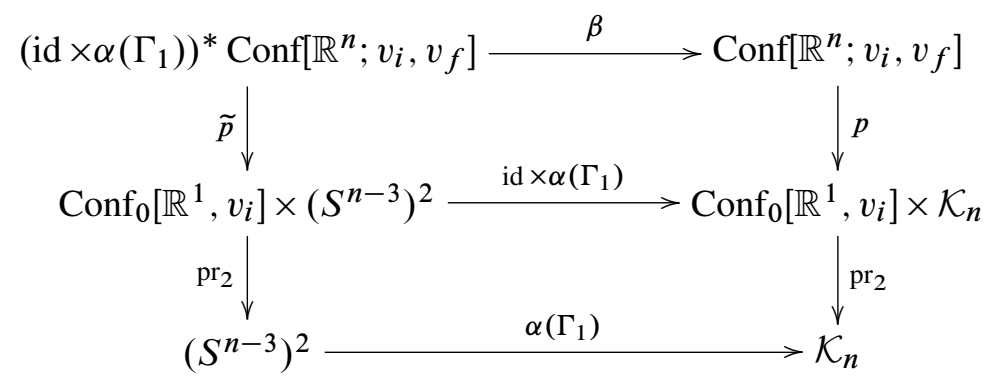

Then

$$
\left\langle I\left(\Gamma_{j}\right), \alpha\left(\Gamma_{1}\right)\right\rangle=\int_{\left(S^{n-3}\right)^{2}}\left(\operatorname{pr}_{2} \circ \widetilde{p}\right)_{*} \beta^{*} \theta_{\Gamma_{j}}=\int_{\operatorname{Conf}_{0}\left[\mathbb{R}^{1}, v_{i}\right] \times\left(S^{n-3}\right)^{2}} \tilde{p}_{*} \beta^{*} \theta_{\Gamma_{j}},
$$


and in this case the integrands are

$$
\theta_{\Gamma_{1}}=\theta_{13} \theta_{24}, \quad \theta_{\Gamma_{2}}=\theta_{14} \theta_{24} \theta_{34}
$$

Recall that the immersion $f$ has the transversal self-intersections $z_{i}=f\left(\xi_{i}\right)=f\left(\xi_{i+2}\right)$, $\xi_{1}<\xi_{2}<\xi_{3}<\xi_{4}$. Let $\varepsilon_{i}>0(i=1,2)$ be sufficiently small numbers which appear in the definition of the resolution of $f$ (Section 2.2) and define the subspace $C=C_{\varepsilon} \subset \operatorname{Conf}_{0}\left[\mathbb{R}^{1}, v_{i}\right]$ by

$$
C_{\epsilon}:=\left\{\begin{array}{l|l}
\left(t_{1}, \ldots, t_{v_{i}}\right) \in \operatorname{Conf}_{0}\left[\mathbb{R}^{1}, v_{i}\right] & \begin{array}{c}
1 \leq \exists i \leq 4,\left|t_{m}-\xi_{i}\right|>\varepsilon_{i} \\
\text { for any } 1 \leq m \leq v_{i}
\end{array}
\end{array}\right\} .
$$

Notice that the complementary set $\operatorname{Conf}_{0}\left[\mathbb{R}^{1}, v_{i}\right] \backslash C$ is the set of configurations such that there is at least one $t_{m}$ near $\xi_{i}, 1 \leq i \leq 4$.

Write $\omega^{(j)}:=\tilde{p}_{*} \beta^{*} \theta_{\Gamma_{j}}$ and

$$
\begin{aligned}
& \omega_{C}^{(j)}:=\int_{C} \omega^{(j)}, \quad \eta_{C}^{(j)}:=\int_{\operatorname{Conf}_{0}\left[\mathbb{R}^{1}, v_{i}\right] \backslash C} \omega^{(j)} . \\
& \left\langle I\left(\Gamma_{j}\right), \alpha\left(\Gamma_{1}\right)\right\rangle=\int_{\left(S^{n-3}\right)^{2}} \omega_{C}^{(j)}+\int_{\left(S^{n-3}\right)^{2}} \eta_{C}^{(j)} .
\end{aligned}
$$

Then

Even if we reduce the "sizes" $\delta_{i}$ of the resolutions of $i$-th self-intersection of the immersion $f$ (see Section 2.2), we still have a homologous cycle $\alpha\left(\Gamma_{1}\right)$, hence the value $\left\langle I(\Gamma), \alpha\left(\Gamma_{1}\right)\right\rangle$ remains unchanged. So we have

$$
\left\langle I(\Gamma), v_{2}\right\rangle=\lim _{\delta_{1}, \delta_{2} \rightarrow 0} \sum_{j=1,2}\left(\int_{\left(S^{n-3}\right)^{2}} \omega_{C}^{(j)}+\int_{\left(S^{n-3}\right)^{2}} \eta_{C}^{(j)}\right) .
$$

But the limit of the integration of $\omega_{C}^{(j)}$ is zero, since on $C$ there is at least one $\xi_{i}$ whose neighborhood does not contain any configuration point $t_{m}$, then the size of the resolution can be reduced to exactly zero at the corresponding doublepoint $z_{k}$ (because collision of configuration points never occur), and the dimension of the cycle decreases.

Thus only the second term, the integration over $\operatorname{Conf}_{0}\left[\mathbb{R}^{1}, v_{i}\right] \backslash C$ contributes to the limit of $\left\langle I(\Gamma), \alpha\left(\Gamma_{1}\right)\right\rangle$. Since there are four $\xi_{i}$ 's, $\operatorname{Conf}_{0}\left[\mathbb{R}^{1}, v_{i}\right] \backslash C \neq \varnothing$ only if $v_{i} \geq 4$. But $\Gamma_{2}$ has only three interval vertices, so cannot contribute to the pairing, while $\Gamma_{1}$ may contribute to the pairing since it has four interval vertices (in general cases, $\operatorname{Conf}_{0}\left[\mathbb{R}^{1}, v_{i}\right] \backslash C \neq \varnothing$ only for the graphs which are chord diagrams). 
So it suffices to compute the limit of

$$
\begin{aligned}
\int_{\left(S^{n-3}\right)^{2}} \eta_{C}^{(1)} & =\int_{\left(\operatorname{Conf}_{0}\left[\mathbb{R}^{1}, 4\right] \backslash C\right) \times\left(S^{n-3}\right)^{2}} \tilde{p}_{*} \beta^{*} \theta_{\Gamma_{1}} \\
& =\int_{\beta \widetilde{p}^{-1}\left\{\left(\operatorname{Conf}_{0}\left[\mathbb{R}^{1}, 4\right] \backslash C\right) \times\left(S^{n-3}\right)^{2}\right\}} \theta_{13} \theta_{24} .
\end{aligned}
$$

Recall from Remark 2.3 the $(n-2)$-sphere $S_{i}$ generated by all the resolution of $z_{i}$, which has the linking number one with the segment $I_{i}(i=1,2)$. The set

$$
\beta \tilde{p}^{-1}\left\{\left(\operatorname{Conf}_{0}\left[\mathbb{R}^{1}, 4\right] \backslash C\right) \times\left(S^{n-3}\right)^{2}\right\}
$$

is precisely the disjoint union $\bigsqcup_{i=1,2} S_{i} \sqcup I_{i}$, and the above integration is

$$
\prod_{i=1,2} \int_{S_{i} \times I_{i}} \varphi_{i}^{*} \operatorname{vol}_{S^{n-1}}
$$

for the Gauss map $\varphi_{i}: S_{i} \times I_{i} \rightarrow S^{n-1}$. Its limit is the product of the linking numbers of $S_{i}$ and $I_{i}(i=1,2)$, thus equal to one. Thus

$$
\left\langle I(\Gamma), \alpha\left(\Gamma_{1}\right)\right\rangle=\lim _{\delta_{i} \rightarrow 0}\left\langle I\left(\Gamma_{1}\right), \alpha\left(\Gamma_{1}\right)\right\rangle= \pm 1 .
$$

\subsection{Nontrivalent graph cocycle}

At present it is not known in general whether the map $I: H^{k, l}\left(\mathcal{D}^{*}\right) \rightarrow H_{\mathrm{DR}}^{(n-3) k+l}\left(\mathcal{K}_{n}\right)$, $l>0$, yields nontrivial cohomology class of $\mathcal{K}_{n}$. But we can see (using work of Salvatore [13], Tourtchine [17] and the author [12]) that, when $n>3$ is odd,

$$
\operatorname{rank} H_{3 n-8}\left(\mathcal{K}_{n}\right)=1 .
$$

So we can expect that $I: H^{3,1}\left(\mathcal{D}^{*}\right) \rightarrow H_{\mathrm{DR}}^{3 n-8}\left(\mathcal{K}_{n}\right)$ might produce a nontrivial cohomology class which is dual to the generator of $H_{3 n-8}\left(\mathcal{K}_{n}, \mathbb{R}\right)$.

It is difficult to compute $H^{3, l}\left(\mathcal{D}^{*}\right)(l \geq 1)$ by hand, but computer calculus tells us the following.

Lemma 3.9 If $n$ is odd, then $H^{3,1}\left(\mathcal{D}^{*}\right) \cong \mathbb{R}$. As a generator we can choose the cochain shown as in Figure 10.

It can be easily seen by a direct computation that the cochain $\Gamma$ in Figure 10 is really a cocycle. It cannot be a coboundary, since $I(\Gamma) \in H^{3 n-8}\left(\mathcal{K}_{n}\right)$ is not zero as we will prove later.

In Figure 10, we omit the labels of the vertices and the orientations of the edges. Unless otherwise indicated, 
- the labels of interval vertices are defined accordingly to the orientation of the line, and

- the orientations of the edges are defined so that the label of the initial vertex of an edge is smaller than that of the terminal one.
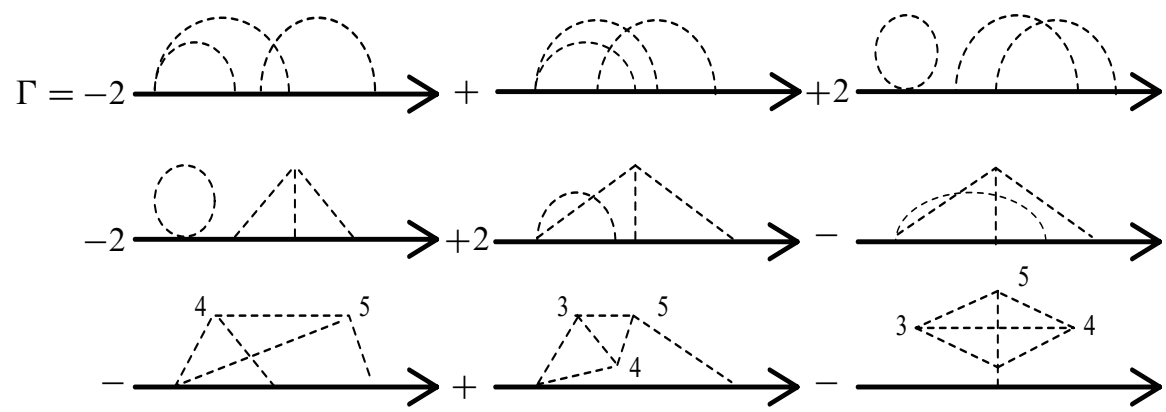

Figure 10: A generator $\Gamma \in H^{3,1}\left(\mathcal{D}^{*}\right)$

Remark 3.10 It can be easily seen that $H^{3, l}\left(\mathcal{D}^{*}\right)=\{0\}, l \geq 4$. The author has not computed $H^{3, l}\left(\mathcal{D}^{*}\right), l=2,3$. But Tourtchine's computation [17, Appendix B] of certain spectral sequence related to $\mathcal{D}^{*}$ suggests that $H^{3, l}\left(\mathcal{D}^{*}\right)$ might be zero for $l=2$, 3. In fact $H^{3,0}\left(\mathcal{D}^{*}\right) \cong \mathbb{R}$ and the Euler characteristic of the complex $\mathcal{D}^{3, *}$ is zero, so rank $H^{3,2}\left(\mathcal{D}^{*}\right)-\operatorname{rank} H^{3,3}\left(\mathcal{D}^{*}\right)=0$. Thus there would be no contradiction even if $H^{3,2}\left(\mathcal{D}^{*}\right)=H^{3,3}\left(\mathcal{D}^{*}\right)=0$. Of course it is not difficult to compute $H^{3, l}\left(\mathcal{D}^{*}\right)$, $l=2,3$, though it would be exhausting.

\section{Evaluation}

Suppose $n>3$ is odd and let $\Gamma \in H^{3,1}\left(\mathcal{D}^{*}\right)$ be the cocycle in Figure 10. Recall $\Lambda \in H_{3 n-8}\left(\mathcal{K}_{n}\right)$ from Section 2.3. The following theorem proves our main result.

Theorem 4.1 The pairing $\langle I(\Gamma), \Lambda\rangle$ is not zero.

Proof We name the nine graphs in Figure $10 \Gamma_{1}, \ldots, \Gamma_{9}$, respectively; so

$$
\Gamma=-2 \Gamma_{1}+\Gamma_{2}+2 \Gamma_{3}-2 \Gamma_{4}+2 \Gamma_{5}-\Gamma_{6}-\Gamma_{7}+\Gamma_{8}-\Gamma_{9} .
$$

First we remark the following fact [5]; the configuration space integral construction explained in Section 3 can be proceeded as long as the volume form of $S^{n-1}$ is 
symmetric, that is, $i^{*} \operatorname{vol}_{S^{n-1}}=-\operatorname{vol}_{S^{n-1}}$ for the antipodal map $i: S^{n-1} \rightarrow S^{n-1}$ (we are assuming $n$ is odd). When $n>4$, the cohomology classes of $\mathcal{K}_{n}$ obtained via the configuration space integrals do not depend on the choice of such symmetric volume forms. So below we use the symmetric volume form whose support is localized in the (sufficiently small) neighborhood of $( \pm 1,0, \ldots, 0) \in S^{n-1} \subset \mathbb{R}^{n}$.

Let $\delta_{i}>0(i=1,2)$ be the "sizes" of resolutions of the self-intersections $z_{i}=f\left(\xi_{i}\right)=$ $f\left(\xi_{i+2}\right)(i=1,2)$ of the immersion $f$ representing $v_{2}$ (see Sections 2.2 and 2.3). We set $\delta_{i}=\varepsilon_{i}^{2}, i=1,2\left(\varepsilon_{i}\right.$ appears in the description of the resolution; see Section 2.2). We will compute the limit $\varepsilon_{i} \rightarrow 0$ of the pairing $\langle I(\Gamma), \Lambda\rangle$. The homology class $[\Lambda]$ is independent of the values $\varepsilon_{i}$, and so is the pairing. But in the limit, as we will prove later, all the graphs $\Gamma_{j}$ except for $\Gamma_{2}$ do not contribute to $\langle I(\Gamma), \Lambda\rangle$ (Lemmas 4.2, 4.3, 4.4 and 4.5), and $\left\langle I\left(\Gamma_{2}\right), \Lambda\right\rangle$ is not zero (Lemmas 4.6 and 4.7).

Lemma 4.2 In the limit, $\left\langle I\left(\Gamma_{j}\right), \Lambda\right\rangle \rightarrow 0$ for $j=7,8,9$.

Proof As in the computation in Section 3.3, only the integration over $\operatorname{Conf}_{0}\left[\mathbb{R}^{1}, v_{i}\right] \backslash C_{\varepsilon}$ contributes to the above pairing in the limit. So the graphs $\Gamma_{j}$ with less than four interval vertices never contribute to the pairing in the limit $\varepsilon_{i} \rightarrow 0$.

Lemma 4.3 In the limit, $\left\langle I\left(\Gamma_{j}\right), \Lambda\right\rangle \rightarrow 0$ for $j=4,5$.

Proof The graphs $\Gamma_{4}$ and $\Gamma_{5}$ have four vertices on the special line. So the corresponding points $\left(t_{1}, \ldots, t_{4}\right)$ is in $\operatorname{Conf}_{0}\left[\mathbb{R}^{1}, 4\right] \backslash C_{\varepsilon}$ if and only if $\left|t_{i}-\xi_{i}\right| \leq \varepsilon_{i}, 1 \leq i \leq 4$. Then in the case of $\Gamma_{4}$, the integrand $\theta_{11}$ is zero since we take the immersion $f$ so that $D_{1} f\left(t_{1}\right)$ with $\left|t_{1}-\xi_{1}\right| \leq \varepsilon_{1}$ cannot be near $( \pm 1,0, \ldots, 0)$, the support of our volume form. In the case of $\Gamma_{5}$, the integrand $\theta_{12}$ also vanishes by similar reason.

Lemma 4.4 In the limit, $\left\langle I\left(\Gamma_{6}\right), \Lambda\right\rangle \rightarrow 0$.

Proof The points $\left(t_{1}, \ldots, t_{4}\right) \in \operatorname{Conf}_{0}\left[\mathbb{R}^{1}, 4\right] \backslash C_{\varepsilon}$ corresponding to interval vertices should be as in the above Lemma. So the integrand $\theta_{15} \theta_{25} \theta_{45}$ vanishes unless the point $x_{5}$ corresponding to the free vertex 5 is "near $( \pm \infty, 0, \ldots, 0)$," since otherwise the images of $\varphi_{i 5} \circ \widetilde{\mathrm{ev}}, i=1,2,4$, cannot be in the support of $\operatorname{vol}_{S^{n-1}}$ simultaneously.

Now we look at two maps $\varphi_{i 5} \circ \widetilde{\mathrm{ev}}, i=2,4$. In the limit $\varepsilon_{2} \rightarrow 0$, the points $f\left(t_{2}\right) \in S_{2}$ and $f\left(t_{4}\right) \in I_{2}$ are very near $\left(S_{2} \approx S^{n-2}\right.$ and the interval $I_{2}$ have been introduced in Remark 2.3), and the free point $x_{5}$ has to be far from them. So the image of the map

$$
\left(\varphi_{25} \circ \widetilde{\mathrm{ev}}\right) \times\left(\varphi_{45} \circ \widetilde{\mathrm{ev}}\right): S_{2} \times I_{2} \longrightarrow\left(S^{n-1}\right)^{2}
$$


is near the diagonal set $\Delta=\left\{(v, v) \in\left(S^{n-1}\right)^{2}\right\}$. More precisely, for any open neighborhood $U$ of $\Delta$, there exists $\epsilon_{0}>0$ such that the image of $\left(\varphi_{25} \circ \widetilde{\mathrm{ev}}\right) \times\left(\varphi_{45} \circ \widetilde{\mathrm{ev}}\right)$ is contained in $U$ for any $\varepsilon_{2}<\varepsilon_{0}$.

Thus on $\operatorname{Conf}_{0}\left[\mathbb{R}^{1}, 4\right] \backslash C_{\varepsilon}$ the integrand $\theta_{45}$ can be written as

$$
\theta_{45}=\left(\varphi_{25} \circ \widetilde{\mathrm{ev}}+\varepsilon^{\prime} \varphi\right)^{*} \operatorname{vol}_{S^{n-1}}
$$

for the Gauss map $\varphi: S_{2} \times I_{2} \rightarrow S^{n-1}$ and some $\varepsilon^{\prime}>0$ such that $\varepsilon^{\prime} \stackrel{\varepsilon_{2} \downarrow 0}{\longrightarrow} 0$. Hence the integrand is

$$
\theta_{13} \theta_{15} \theta_{25} \theta_{45}=\varepsilon^{\prime} \theta_{13} \theta_{15} \theta_{25} \varphi^{*}\left(\operatorname{vol}_{S^{n-1}}\right)
$$

and its integration converges to zero as $\varepsilon_{2} \rightarrow 0$.

Lemma 4.5 In the limit, $\left\langle I\left(\Gamma_{j}\right), \Lambda\right\rangle \rightarrow 0$ for $j=1,3$.

Proof First we prove $\left\langle I\left(\Gamma_{1}\right), \Lambda\right\rangle \rightarrow 0$; the integrand $\theta_{12}$ is not zero only if $t \in$ $\operatorname{Conf}_{0}\left[\mathbb{R}^{1}, 4\right] \backslash C$ is such that $t_{1}$ is near $\xi_{1}$ and $t_{2}$ is near $\xi_{3}$. But then no other $t_{i}$ can be near $\xi_{2}$.

$\left\langle I\left(\Gamma_{3}\right), \Lambda\right\rangle \rightarrow 0$ since if $t \in \operatorname{Conf}_{0}\left[\mathbb{R}^{1}, 4\right] \backslash C$ then $t_{1} \leq \xi_{1}$ and thus $\theta_{11}=D_{1}^{*}$ vol is always zero by our choice of $f$.

Lemma 4.6 The limit of $\left\langle I\left(\Gamma_{2}\right), \Lambda\right\rangle$ is not zero.

Proof The integrand $\theta_{13} \theta_{14} \theta_{25}$ does not vanish only if the $t \in \operatorname{Conf}_{0}\left(\mathbb{R}^{1}, 5\right) \backslash C$ is such that $t_{1}$ is near $\xi_{1}, t_{3}, t_{4}$ are near $\xi_{3}, t_{2}$ is near $\xi_{2}$ and $t_{5}$ is near $\xi_{4}$, and the images of direction maps $\varphi_{i j} \circ \widetilde{\mathrm{ev}}((i, j)=(1,3),(1,4),(2,5))$ are "near" $( \pm 1,0, \ldots, 0)$ (see Figure 11).

Integration with respect to $t_{2}, t_{5}$ and $u_{2} \in S^{n-3}$ is the linking number, and hence equals to one (Remark 2.3). So it remains to compute the integration with respect to $t_{1}, t_{3}, t_{4}$ and $u_{1} \in S^{n-3}$. We reformulate the situation around the resolved singular point $z_{1}$ (which is indicated in Figures 7 and 11) as follows (see Figure 12):

- a point $P_{1}$ (corresponding to $\left.f\left(t_{1}\right)\right)$ is on

$$
M:=\left\{x_{1}^{2}+\cdots+x_{n-2}^{2}+x_{n}^{2}=1, x_{n-1}=0\right\}
$$

(this sphere corresponds to $S_{1} \approx S^{n-2}$ introduced in Section 3.3),

- two points $\left(P_{4}, P_{3}\right) \in \operatorname{Conf}_{0}\left[\mathbb{R}^{1}, 2\right]$ (corresponding to $\left.f\left(t_{4}\right), f\left(t_{3}\right)\right)$ are on the $x_{n-1}$-axis (corresponding to the interval $I_{1}$ ), and

- the frame $e[s, u] \in S O(n-1)\left([s, u] \in \Sigma S^{n-3}\right)$ acts on $\mathbb{R}^{n}$, fixing the $x_{n}$-axis. 


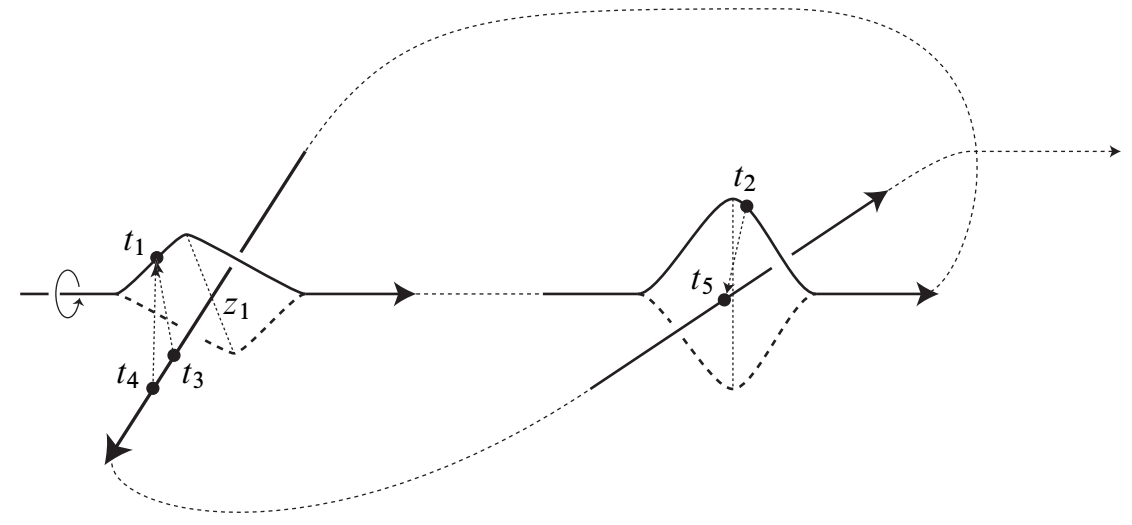

Figure 11: A configuration $\left(t_{1}, \ldots, t_{5}\right)$ which contributes to $\left\langle I\left(\Gamma_{2}\right), \Lambda\right\rangle$

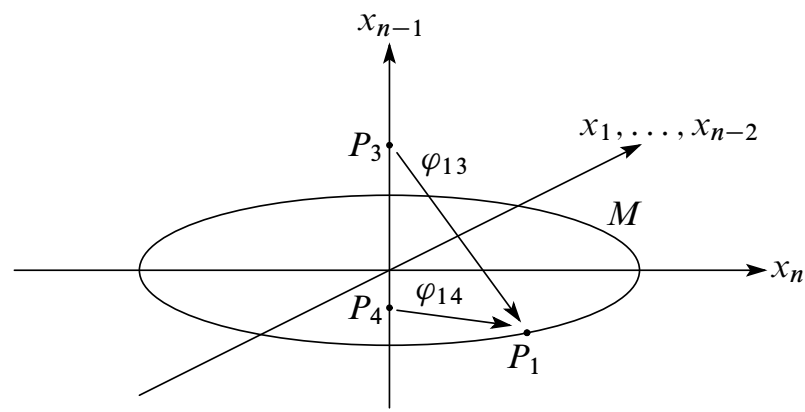

Figure 12: Integration around $z_{1}$

If we define

$$
F: N \longrightarrow\left(S^{n-1}\right)^{2} \quad\left(N:=\Sigma S^{n-3} \times S^{n-2} \times \operatorname{Conf}_{0}\left[\mathbb{R}^{1}, 2\right]\right)
$$

by

$$
\begin{aligned}
F\left([s, u], P_{1},\left(P_{4}, P_{3}\right)\right): & =\left(\varphi_{13}, \varphi_{14}\right)\left(e[s, u] P_{i}\right)_{i=1,3,4} \\
& =\left(e[s, u] \frac{P_{1}-P_{3}}{\left|P_{1}-P_{3}\right|}, e[s, u] \frac{P_{1}-P_{4}}{\left|P_{1}-P_{4}\right|}\right),
\end{aligned}
$$

then our aim is to compute the integral

$$
\int_{N} F^{*}\left(\operatorname{vol}_{\left(S^{n-1}\right)^{2}}\right)
$$


where $\operatorname{vol}_{\left(S^{n-1}\right)^{2}}=\operatorname{pr}_{1}^{*} \operatorname{vol}_{S^{n-1}} \wedge \operatorname{pr}_{2}^{*} \operatorname{vol}_{S^{n-1}}$ is a top form of $\left(S^{n-1}\right)^{2}$ (remember the support of $\operatorname{vol}_{S^{n-1}}$ is localized in the neighborhood of $\left.( \pm 1,0, \ldots, 0)\right)$.

The map $F$ has its image in

$$
A:=\left\{(x, y) \in\left(S^{n-1}\right)^{2} \mid x_{n} y_{n} \geq 0\right\}
$$

where $x_{n}$ is the $n$-th coordinate of $x \in S^{n-1} \subset \mathbb{R}^{n}$. In fact, as we will prove in Lemma 4.7, the map $F$ is two-fold covering on Int $A$ branched on the diagonal $\Delta=\{(v, v) \in$ $\left.\left(S^{n-1}\right)^{2}\right\}$, thus the image of $F$ covers the half of the support of $\operatorname{vol}_{\left(S^{n-1}\right)^{2}}$ twice. Moreover, for any $p, q \in F^{-1}($ Int $A \backslash \Delta)$ with $F(p)=F(q)$, the map $G: T_{p} N \rightarrow T_{q} N$ defined by the commutative diagram

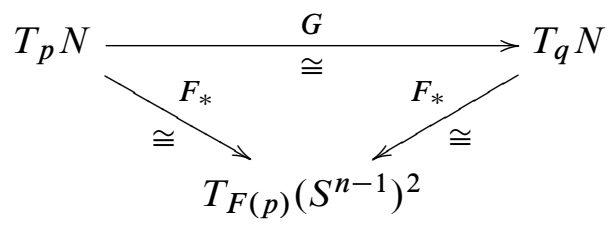

is an isomorphism of oriented tangent spaces for suitable orientations of $N$ and $\left(S^{n-1}\right)^{2}$. Thus the limit of the above integral is $\pm 1 / 2 \times 2= \pm 1$.

Lemma 4.7 $\left.F\right|_{\text {Int } N}$ is two-fold smooth covering onto Int $A \backslash \Delta$ and $G$ is an orientation preserving map.

Proof We denote by $(v)_{n}$ the $n$-th coordinate of $v \in \mathbb{R}^{n}$. We will show that for any $\left(v_{3}, v_{4}\right) \in \operatorname{Int} A \backslash \Delta$ (then $\left.\left(v_{3}\right)_{n}\left(v_{4}\right)_{n}>0\right)$, we can find $\left([s, u], P_{i}\right)$ so that $F\left([s, u], P_{i}\right)=$ $\left(v_{3}, v_{4}\right)$, that is,

$$
e[s, u] \frac{P_{1}-P_{i}}{\left|P_{1}-P_{i}\right|}=v_{i}, \quad i=3,4 .
$$

Consider the 2-plane $H\left(v_{3}, v_{4}\right) \subset \mathbb{R}^{n}$ spanned by two vectors $v_{3}, v_{4}$. Then the intersection

$$
l\left(v_{3}, v_{4}\right):=H\left(v_{3}, v_{4}\right) \cap\left\{x_{n}=0\right\}
$$

is a one-dimensional linear subspace of $\left\{x_{n}=0\right\}$.

Since $\left([s, u], P_{i}\right)$ should satisfy $(4-1)$, the points $e[s, u] P_{i}(i=3,4)$ should be on $l\left(v_{3}, v_{4}\right)$ and $e[s, u] P_{1}$ should be on $H\left(v_{3}, v_{4}\right) \cap e[s, u] M$. So the frame $(s, u)$ should transpose $x_{n-1}$-axis to $l\left(v_{3}, v_{4}\right)$. There are two such frames, namely, $[s, u]$ and $[-s,-u]$ for some $[s, u] \in \Sigma S^{n-3}$. We have $e[s, u] M=e[-s,-u] M$, and this sphere intersects with $H\left(v_{3}, v_{4}\right)$ at two points. One has positive $n$-th coordinate and the other has negative one. When $\left(v_{3}\right)_{n}>0$ (resp. $\left.\left(v_{3}\right)_{n}<0\right)$, we choose positive (resp. 
negative) one and name it $e[s, u] P_{1}$. Then $P_{3}$ and $P_{4}$ are determined uniquely so that $\varphi_{1 i}\left(P_{1}, P_{3}, P_{4}\right)=e[s, u]^{-1} v_{i}$.

Thus we have two points $\left( \pm[s, u], P_{i}\right)$ which are mapped to $\left(v_{3}, v_{4}\right) \in\left(S^{n-1}\right)^{2}$ via $F$. The map $F$ is clearly smooth. The above arguments show that $F^{-1}$ is also smooth, hence $\left.F\right|_{\text {Int } N}$ is locally diffeomorphic two-fold covering.

The map $G$ is orientation preserving, since it is essentially the antipodal map $\Sigma S^{n-3} \rightarrow$ $\Sigma S^{n-3}$ and it preserves orientation (we assume $n$ is odd).

Remark 4.8 In general, nothing is known about $H^{k, 1}\left(\mathcal{D}^{*}\right), k \geq 4$. But anyway suppose we have $\Gamma=\sum a_{i} \Gamma_{i} \in H^{k, 1}\left(\mathcal{D}^{*}\right)$. Let $\Gamma^{\prime}$ be a chord diagram with $(k-1)$ chords. Then, in a similar way as above, we can compute the pairing $\left\langle I(\Gamma), r_{*} \lambda\left(\alpha\left(\Gamma^{\prime}\right), e\right)\right\rangle$; choose an immersion $f$ representing $\alpha\left(\Gamma^{\prime}\right)$ so that almost all of the image of $f$ lies in $x_{n-1} x_{n}$-axis. We proceed the configuration space integral construction by using the symmetric volume form of $S^{n-1}$ whose support is localized in the neighborhood of $( \pm 1,0, \ldots, 0)$. Let $\delta_{i}>0$ be the "size" of the $i$-th resolution of the immersion $f$. Then, in the limit $\delta_{i} \rightarrow 0(1 \leq i \leq k-1)$, only the graphs $\Gamma_{i}$ obtained from $\Gamma^{\prime}$ with one of its chord "doubled" contribute to the pairing (see Figure 13).

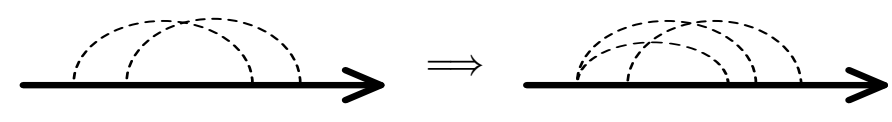

Figure 13: Doubling operation

\section{References}

[1] S Axelrod, I M Singer, Chern-Simons perturbation theory. II, J. Differential Geom. 39 (1994) 173-213 MR1258919

[2] R Bott, C Taubes, On the self-linking of knots, J. Math. Phys. 35 (1994) 5247-5287 MR1295465Topology and physics

[3] R Budney, Little cubes and long knots, Topology 46 (2007) 1-27 MR2288724

[4] R Budney, F Cohen, On the homology of the space of knots arXiv: math.GT/0504206

[5] A S Cattaneo, P Cotta-Ramusino, R Longoni, Configuration spaces and Vassiliev classes in any dimension, Algebr. Geom. Topol. 2 (2002) 949-1000 MR1936977

[6] F R Cohen, The homology of $\mathcal{C}_{n+1}-$ space, $n \geq 0$, from: "The homology of iterated loop spaces", Lecture Notes in Math. 533, Springer, Berlin (1976) vii+490 MR0436146 
[7] T Kohno, Vassiliev invariants and de Rham complex on the space of knots, from: "Symplectic geometry and quantization (Sanda and Yokohama, 1993)", Contemp. Math. 179, Amer. Math. Soc. (1994) 123-138 MR1319605

[8] P Lambrechts, V Tourtchine, I Volić, The rational homology of spaces of knots in codimension $>2$ arXiv:math.AT/0703649

[9] R Longoni, Nontrivial classes in $H^{*}\left(\operatorname{Imb}\left(S^{1}, \mathbb{R}^{n}\right)\right)$ from nontrivalent graph cocycles, Int. J. Geom. Methods Mod. Phys. 1 (2004) 639-650 MR2095442

[10] J P May, The geometry of iterated loop spaces, Lecture Notes in Math. 271, Springer, Berlin (1972) MR0420610

[11] J E McClure, J H Smith, Cosimplicial objects and little $n$-cubes. I, Amer. J. Math. 126 (2004) 1109-1153 MR2089084

[12] K Sakai, Poisson structures on the homology of the space of knots, from: "Groups, homotopy and configuration spaces (Tokyo 2005)", (N Iwase, T Kohno, R Levi, D Tamaki, J Wu, editors), Geom. Topol. Monogr. 13 (2008) 463-482

[13] P Salvatore, Knots, operads, and double loop spaces, Int. Math. Res. Not. (2006) Art. ID 13628, 22 MR2276349

[14] D P Sinha, The topology of spaces of knots arXiv:math. AT/0202287

[15] D P Sinha, Manifold-theoretic compactifications of configuration spaces, Selecta Math. (N.S.) 10 (2004) 391-428 MR2099074

[16] DP Sinha, Operads and knot spaces, J. Amer. Math. Soc. 19 (2006) 461-486 MR2188133

[17] V Tourtchine, On the other side of the bialgebra of chord diagrams, J. Knot Theory Ramifications 16 (2007) 575-629 MR2333307

[18] I Volić, A survey of Bott-Taubes integration, J. Knot Theory Ramifications 16 (2007) 1-42 MR2300426

Graduate School of Mathematical Sciences, The University of Tokyo 3-8-1 Komaba, Meguro, Tokyo 153-8914, Japan

ksakai@ms.u-tokyo.ac.jp

Received: 31 December 2007 Revised: 18 July 2008 